\title{
Turning a Liability into an Asset: Radiation Damage as a Cytochemical Marker.
}

\author{
Naiqian Cheng ${ }^{1}$, Weimin $\mathrm{Wu}^{1}$, Norman R. Watts ${ }^{2}$, Juan Fontana ${ }^{1}$ and Alasdair C. Steven ${ }^{1}$ \\ ${ }^{1}$ Laboratory of Structural Biology Research \& ${ }^{2}$ Protein Expression Laboratory, National Institute of \\ Arthritis, Musculoskeletal and Skin Diseases, National Institutes of Health, Bethesda, U.S.A.
}

An essential step in the structural analysis of large macromolecular complexes by cryo-electron microscopy involves mapping the various components - proteins and nucleic acids, as the case may be in three-dimensional density maps. For surface-exposed components, a number of approaches are possible, including antibody labeling with Fab fragments or attaching heavy metal clusters at biochemically defined sites. However, components that are buried deep within a complex are not accessible to surface probes and require alternative approaches. The problem is particularly acute for the nucleocapsids of large viruses in which essential proteins - scaffolding proteins or polymerases, for example - may be embedded within the densely packaged viral genomes. We have recently discovered that such proteins are extremely sensitive to radiation damage and, in vitrified specimens, they "bubble" prematurely, i.e. when exposed to electron doses that leave proteins which are not so confined only slightly blurred in micrographs [1]. The bubbles are of hydrogen gas at high pressure [2, 3]. Nucleic acid does not bubble under the same conditions; nor do free-standing proteins, i.e. ones that are in contact with or close to the frozen buffer: apparently, the surrounding nucleic acid impedes the diffusion of radiolytic products from their sites of origin so that they build up sooner to concentrations at which bubbles nucleate and then grow [4]. These properties make it possible to distinguish protein from DNA. An initial low-dose micrograph is used from structural analysis. Subsequent exposures of the same field elicit bubbling that reveals the locations of internal proteins embedded in DNA. Nucleic acids appear are perceived as dense bubbling-resistant material. We call this approach "bubblegram imaging" [1].

We have used a CM200-FEG electron microscope (FEI) with a Gatan 626 cryo-holder maintaining a specimen temperature of $\sim-180^{\circ} \mathrm{C}$, operated at $120 \mathrm{keV}$ and $38,000 \mathrm{x}$ magnification to record dose series of up to 9 exposures. Each 1 -second exposure corresponds to a dose of $17 \mathrm{e}^{-} / \AA^{2}$. The micrographs analyzed were recorded on film, digitized, and the data were processed using Bsoft and EMAN2.

We first applied this technique to the giant bacteriophage phiKZ and detected a cylindrical "inner body", $\sim 105 \mathrm{~nm}$ long and $24 \mathrm{~nm}$ in diameter with an estimated mass of $\sim 15 \mathrm{MDa}$ [1]. The inner body is invisible in low-dose micrographs but shows up as a column of bubbles in high-dose micrographs. Interestingly, the inner body, which has 6-fold rotational symmetry, is anchored not on the portal protein, a 12-membered ring located at one of the 5-fold vertices of the icosahedral capsid, but on a neighboring symmetry-matched hexamer of capsid protein. The inner body may serve as a spindle for organizing genomic DNA. Next, the technique was extended to the smaller bacteriophage T7 (60 nm diameter vs. 145nm) which has a barrel-shaped protein core, $25 \mathrm{~nm}$ long by $20 \mathrm{~nm}$ in diameter mounted coaxially on the portal vertex [4] - Figure 1. In this case, high-dose micrographs were also reconstructed, revealing the development of a bipartite gas cloud. This analysis led to a more detailed interpretation of the core structure and identified a bubbling-resistant rod of density, apparently representing a linear segment of DNA poised for ejection into a host cell [4].

While many aspects of bubblegram imaging remain to be explored, we have so far made the following method-related observations. (1) The onset of bubbling depends on the interval between exposures. With $10 \mathrm{sec}$ between exposures, it starts in the third exposure (cumulative dose $\sim 50 \mathrm{e}^{-} / \AA^{2}$ ); with 10 min 
between exposures, it starts in the fifth exposure. (2) The dose threshold for bubbling and the size of the bubbles depends on the amount of protein in a given complex. (3) Bubbling varies stochastically from particle to particle: in each copy of large protein complex, bubbles nucleate at a few sites, then grow and merge. (4) Susceptibility to bubbling appears to depend to some extent on protein type (amino acid composition). (5) Preliminary results indicate that RNA-protein complexes exhibit bubbling behavior similar to that of DNA-protein complexes.

\section{References:}

[1] W Wu et al., Science 335 (2012), p. 182.

[2] R D Leapman and S Sun, Ultramicroscopy 59(1995), p.71.

[3] A Meents et al., Proc Nat'l Acad Sci USA 107 (2010), p.1094.

[4] N Cheng et al., J Struct Biol (2013) doi: 10.1016/j.jsb.2013.12.004.

[5] This research was supported by the Intramural Research Program of NIAMS.
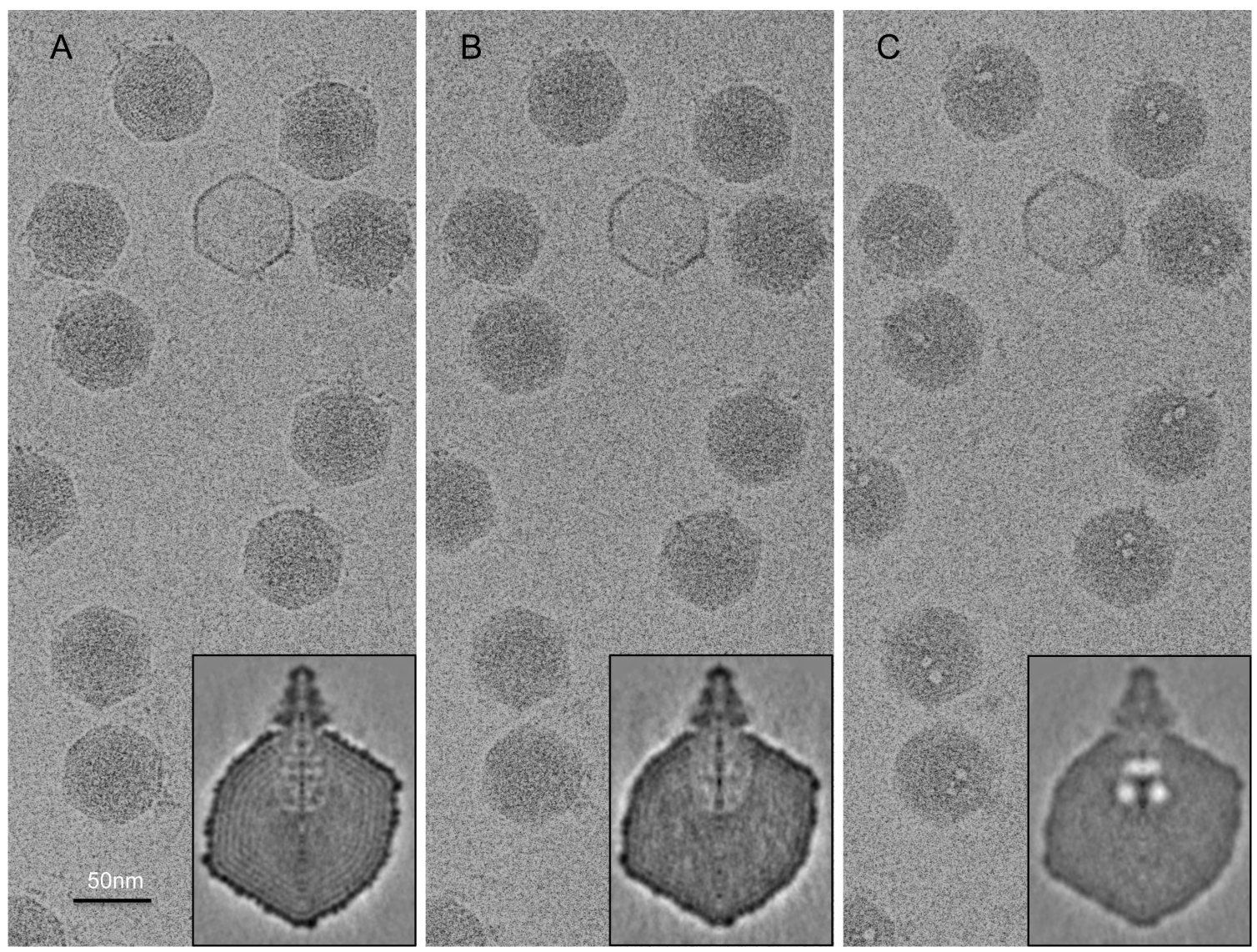

Figure 1. Panels A, B and C show, respectively, the $1^{\text {st }}, 3^{\text {rd }}$ and $5^{\text {th }}$ members of a dose series of cryoelectron micrographs of a field of $\mathrm{T} 7$ virions. Inset at bottom right of each panel is a central sagittal section of the asymmetric reconstruction, i.e. a reconstruction in which the only symmetry imposed was 5-fold symmetry about the portal axis (vertical). In B, the virion images and the reconstruction are blurred relative to $\mathrm{A}$. In $\mathrm{C}, 2-4$ bubbles are visible in each virion and a developing gas cloud (white) is seen in the reconstruction. 